\title{
The Three Dimensional Numerical Simulation of Vertical Jet in Waves
}

\author{
L. R. Yuan*
}

Occan Engineering Research Conter, School of Engineering, Sun Yat-sen University, Guangzhou 510275, China

Email: yuanlr@mial.sysu.edu.cn

\begin{abstract}
A three dimensional numerical model of vertical turbulent jet in waves is established by using the nonlinear $k-\varepsilon$ turbulence model with VOF method. And a lot of numerical experiments with different wave parameters are carried out. Based on the numerical experiments results, the paper put emphases upon the analysis of the relations between the distributions of the pollutant concentration and water waves' parameters in the jet initial entrainment.

Instantaneous velocity field and concentration field show that the jet axial line deflects with wave periodically and the mixing is stronger than that in static water. Wave period averaged velocity field and concentration field in waves display distinct differences with in static water. Based on the comparison of vertical velocity and concentration attenuation characters among different wave length and wave height cases, it is discovered that the effect of wave on jet dilution depends on the ratio of the wave trace amplitude to the jet expanded half width along the water depth.
\end{abstract}

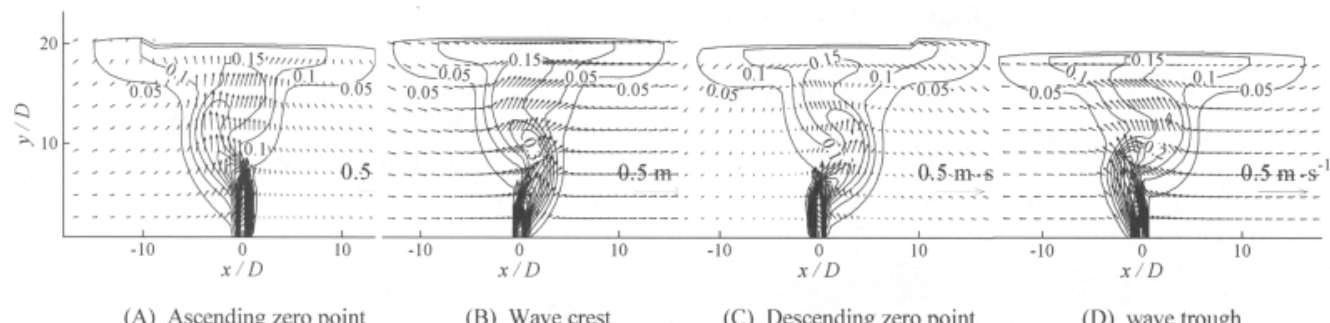

(A) Ascending zero point

(B) Wave crest

(C) Descending zero point

(D) wave trough

Figure: Velocity and concentration field on different wave phases

\section{REFERENCES}

1. Chin D A. Influence of surface waves on outfall dilution. Journal of Hydraulic Engineering, 1987; 113(8): 1006-1018

2. Koole R, Swan C. Measurements of a 2-d non-buoyant jet in a wave environment. Coastal Engineering, 1994; 24(2): 151-169

3. Michele M. Behavior of Non-buoyant Jets in a Wave Environment. Journal of Hydraulic Engineering, 2004; 130(7): 704-717

4. Chen C J, Jaw S Y. Fundamentals of Turbulence Modeling. Washington, DC: Taylor \& Francis, 1998 